\title{
Valoración económica del servicio hídrico de la quebrada San Alberto, Parque Nacional Yanachaga Chemillen
}

\author{
Economic assessment of the water service at the stream San Alberto, Yanachaga Chemillén \\ National Park
}

Roger Alfredo Loyola Gonzáles ${ }^{1}$

\begin{abstract}
Resumen
En el presente estudio se ha determinado el valor económico del servicio hídrico que proviene del Parque Nacional Yanachaga-Chemillén a través de la Quebrada San Alberto. Para ello se utilizó un método de preferencias declaradas, Experimentos de Elección, el mismo que fue aplicado sobre la población de Oxapampa. Los resultados señalan que la disposición a pagar por este servicio es equivalente a S/. 16,66, el cual resultó ser más significativo que las otras variables consideradas.
\end{abstract}

Palabras clave: Yanachaga-Chemillén; servicio hídrico; parque nacional.

\begin{abstract}
The study determined the economic value of the water service that comes from the National Park Yanachaga Chemillén through the Quebrada San Alberto. We used a method of stated preference choice experiments, the same that was applied to the population of Oxapampa. The results showed that willingness to pay for this service is equivalent to $\mathrm{S} / 16,66$, which was more significant than the other variables considered.
\end{abstract}

Keywords: Yanachaga-Chemillén; water service; national park.

\section{Introducción}

Los instrumentos económicos relacionados al ambiente han sido utilizados en el mundo en los últimos años en la prevención de la contaminación y la conservación de los ecosistemas (United Nations Environment ProgrammeUNEP, 2004). La mayor parte de ellos tratan de prevenir externalidades ambientales negativas por medio de impuestos, permisos de emisión transables u otras herramientas de política económica. En este sentido, la valoración económica ambiental puede proporcionar información relevante sobre la importancia económica que los bienes del ambiente tienen para la sociedad a través de la cuantificación de los beneficios que estos generan. Dichos valores serán útiles en la medida que podrían servir de base para la generación adecuada de los instrumentos pertinentes.

En el caso del Perú, especialmente en las tarifas de agua, estas se determinan considerando el costo en que se incurre por hacer disponible este recurso a los consumidores. Sin embargo, esta forma de hacer el cálculo es económicamente ineficiente porque, por un lado, alienta malos comportamientos dado que no representan la importancia que tiene el recurso para las personas y, por otro, porque estas tarifas no permiten atender a nuevos usuarios ya que solo se reciben ingresos de corto plazo, dejando poca maniobrabilidad para nuevas instalaciones o para mejoras de las actuales.

Estas consideraciones suceden en el caso de Oxapampa, donde además de los problemas mencionados, se tiene una tarifa plana para los diferentes usuarios, lo cual agrega un sistema de perversidad mayor, dado que en este tipo de tarifas los usuarios que menos consumen terminan financiando a quien más consume.

En este sentido, el propósito del presente estudio es determinar el valor de la disposición a pagar por parte de la población de Oxapampa por el servicio ambiental hídrico de la quebrada San Alberto, a través de técnicas de preferencias declaradas, con el empleo de la metodología de los Experimentos de Elección (EE). Para ello, se realizaron encuestas en las que se presentaron mercados hipotéticos para los servicios: hídrico, del bosque y de cortes de agua, además de una tarifa adicional al pago mensual por el servicio de agua potable que realizan.

\section{Materiales y métodos \\ Marco conceptual}

Según Colombo (2004), los EE constituyen una aplicación de la teoría del valor de Lancaster asociada a la teoría de la utilidad aleatoria según la cual, a partir de la elección de una combinación, se estima la importancia implícita de 
los atributos que la describen. Como mencionan Hanley el al. (1998), a los entrevistados se les da a escoger entre diferentes canastas de bienes ambientales, los mismos que son descritos en términos de sus atributos y los niveles que representan.

El individuo representativo tiene una función de utilidad de la siguiente forma:

$U_{\text {in }}=U\left(Z_{\dot{n}}, S_{n}\right)$

donde a cualquier individuo $\mathrm{n}$ se le puede asociar un nivel de utilidad relacionado con el bien ambiental i. Este bien i puede ser elegido en comparación con otra opción j si $U_{i}$ $>\mathrm{U}_{\mathrm{j}}$. La utilidad derivada de cualquier elección depende de sus atributos Z. Estos atributos pueden ser vistos de forma diferente por diversos agentes, cuyas características socioeconómicas $\mathrm{S}$, deberán afectar también la utilidad. Esta función de utilidad puede ser representada por dos partes, una determinística y observable y otra aleatoria y no observable. La ecuación (1) puede ser escrita como:

$U_{i n}=V\left(Z_{i n}, S_{n}\right)+\varepsilon\left(Z_{i n}, S_{n}\right)$

La probabilidad que el individuo n escoja la opción i en vez que la $\mathrm{j}$ está dada por:

$\operatorname{Pr} o b(i / C)=\operatorname{Pr} o b\left\{V_{i n}+\varepsilon_{i n}>V_{j n}+\varepsilon_{j n}, \forall i, j \in C\right\}$

Siendo $\mathrm{C}$ el conjunto completo de elecciones. Para poder realizar las estimaciones hay que realizar suposiciones sobre la forma cómo se distribuyen los términos del error. Generalmente, se asume que estos se comportan según la distribución de Gumbel, por tanto, la probabilidad de escoger i está dada por:

$\operatorname{Pr} o b(i)=\frac{\exp ^{v v_{i}}}{\sum_{j \in C} \exp ^{v v_{j}}}$

donde $\mu$ es un parámetro escalar el cual se asume sea igual a 1, lo que implica que la variancia del error es constante. Como $\mu$ tiende al infinito, el modelo se vuelve determinístico; esta ecuación es estimada por una regresión multinomial logit, la cual asume que las elecciones sean consistentes con la propiedad de la independencia de las alternativas irrelevantes, la cual establece que para cualquier individuo el ratio de las probabilidades de elección de dos alternativas no es afectado por las utilidades sistemáticas de otras alternativas.

Si $V\left(\right.$.) es lineal tal que $V=\beta\left(X_{n}\right)$, donde $X$ es un vector de atributos observables y $\beta$ un vector de parámetros a ser estimados, por lo que el modelo se vuelve en:

$$
\operatorname{Pr} o b(i)=\frac{e^{.-v \beta\left(x_{i n} \cdot x_{j n}\right)}}{1+e^{-v \beta\left(x_{i n}-x_{j n}\right)}}
$$

Dado que los modelos de EE comparten el mismo marco conceptual de utilidad aleatoria que los modelos dicotómicos de los patrones de valoración contingente, sus estimaciones del bienestar son comparables.

Hensher et al. (2005) analizaron, a través de una serie de experimentos de elección declarados y modelos logit mixtos, el establecimiento de la disponibilidad a pagar para evitar interrupciones en el servicio de agua y derrames de aguas servidas, diferenciadas por la frecuencia, el tiempo y la duración de estos eventos. La encuesta fue realizada en dos partes: una entrevista inicial de base y la de los experimentos de elección. Se presentaron dos sets de experimentos, uno relativo al servicio de agua potable y el otro para el desagüe. Cada experimento consideraba dos opciones de servicio con sus descripciones y al encuestado se le preguntaba por la opción que prefería. Los resultados confirman que los niveles de los servicios de agua y alcantarillado son importantes para los consumidores.

Hanley et al.2006) determinaron los valores que la gente asigna a las mejoras para los cuerpos de agua, según el Marco Directivo del Agua de la Unión Europea. Se consideraron tres atributos para representar el concepto de "nivel ecológico bueno": la vida acuática incluyendo peces, plantas e invertebrados; la estética, la cual representa la cantidad de basura en el río y la calidad de las riberas, tanto en términos de vegetación como de erosión.

Hatton et al. (2005) exploran el uso de Choice Modelling para estimar precios implícitos asociados con los atributos de la oferta de agua potable. Los resultados de los modelos multinomial (Mulinomial Logit-MNL) y de los parámetros aleatorios del logit (Random Parameter Logit-RPL) muestran que los incrementos en las facturaciones anuales del agua y la frecuencia de futuras interrupciones eran los atributos más importantes.

\section{Descripción del lugar}

El estudio fue realizado en la cuenca del río San Alberto, que se encuentra en la zona de amortiguamiento del Parque Nacional Yanachaga-Chemillén (PNYCh). Es de esta cuenca que la EPS "Selva Central” S.A. (EPS) abastece de agua a la población de Oxapampa, en el departamento de Pasco. La mayoría de los usuarios paga una tarifa plana por mes que es de S/. 8,90 para uso doméstico y de S/. 14,40 para uso comercial. Pocos clientes tienen medidores.

El agua que proviene del parque es de buena calidad; sin embargo, en la zona de amortiguamiento del PNYCh, la actividad ganadera y la producción agrícola, especialmente de rocoto (Capsicum pubescens), granadilla (Passiflora spp.), zapallo (Cucurbita maxima), el maíz (Zea mays) y otras especies, ejercen presión sobre las aguas del río. Estas actividades causan la erosión severa del suelo, conducen a la contaminación del río con bacterias patógenas (coliformes fecales) y agroquímicos, lo que incrementa los costos en la planta de tratamiento de aguas y causa interrupciones del servicio de agua potable.

Las interrupciones del servicio del agua ocurren en gran parte debido a la existencia de sedimentos en San Alberto. La planta de tratamiento puede trabajar adecuadamente con una turbiedad de 30 NTU, alcanzando niveles más altos durante épocas de precipitación pesada o alta. Una precipitación de $15 \mathrm{~mm}$ por un tiempo de dos horas es suficiente para causar deslizamientos de lodo o la erosión severa de los bancos de arena y limo por la corriente de agua del río; además, la precipitación de solamente $4 \mathrm{~mm}$ por hora es bastante para causar un aumento en la turbiedad. El cierre del uso del agua del San Alberto conduce a una 
interrupción del servicio que dura normalmente de 6 a 10 horas, tiempo después del cual el nivel de turbiedad del agua de la quebrada disminuye y el agua del río San Alberto puede ser utilizada otra vez. El reservorio tiene una capacidad de $700 \mathrm{~m}^{3}$ que se llena en un tiempo aproximado de cuatro horas, distribuyéndose en un período de 1,5 horas y se repite el ciclo las veces que se requiera. El número promedio de interrupciones que suceden en una estación húmeda o una estación seca, según la administración de la EPS, se muestra en la Tabla 1, la misma que está expresada en días. En este caso, el 2003 se construyó un sedimentador primario y en el 2004 un filtro.

Tabla 1. Número promedio de interrupciones por día del servicio de agua potable en el distrito de Oxapampa

\begin{tabular}{|c|c|c|}
\hline Años & Estación lluviosa & Estación seca \\
\hline 2000 & 20 & 6 \\
\hline 2001 & 23 & 9 \\
\hline 2002 & 17 & 5 \\
\hline 2003 & 27 & 11 \\
\hline 2004 & 3 & 3 \\
\hline 2005 & 4 & 4 \\
\hline 2006 & 57 & \\
\hline Fuente: EPS Oxapampa.
\end{tabular}

\section{Métodología}

Inicialmente, se realizó un sondeo preliminar en Oxapampa sobre la disponibilidad a pagar (DAP) por el servicio ambiental hídrico que ofrece la quebrada San Alberto, a través de una encuesta piloto. Se hicieron preguntas sobre los problemas ambientales más importantes, relacionados con la quebrada San Alberto, donde se identificó que el problema de la tala, quema y destrucción de bosques para fines de agricultura, la contaminación de las aguas del río San Alberto por los ganaderos y pobladores del lugar, así como el problema de la potabilización y abastecimiento del agua son las actividades que más impacto presentaron. De aquí se obtuvieron los alcances que se emplearían en la encuesta definitiva S/. 2,00, S/. 4,00, S/. 6,00 y S/. 8,00. Se definieron como variables dependientes de la encuesta las siguientes:

- Cortes (interrupciones de servicio de agua potable) considerados a tres niveles: 10 horas de corte promedio/ día - 30 días por año (nivel 1); 4 - 10 horas de corte promedio/día - 15 días por año (nivel 2) y Nunca (nivel $3)$.

- Agua (cualidad del agua del río), considerando 2 niveles: río contaminado (nivel 1) y río sin contaminar (nivel 2).

- Quemas (protección de la cuenca-quemas del bosque), considerando 3 niveles: Mismo número de quemas que hoy (nivel 1), $50 \%$ de las quemas que hoy se realizan (nivel 2) y No se realizan quemas (nivel 3).

- Tarifa (para agua potable mensual) que considera las propuestas por la población para el pago ambiental, obtenidas del sondeo preliminar.
Se consideraron variables sociodemográficas en la encuesta con la finalidad de analizar la consistencia de los resultados como: visitas a la Quebrada San Alberto, calidad del agua San Alberto, instrucción, ingreso, ocupación, edad, años viviendo en el área, número de personas viviendo en la casa, número de personas menores de 18 años de edad y género.

\section{Determinación de la muestra respectiva}

Durante el estudio, la EPS informó que se contaba con 1.400 conexiones domésticas y 110 comerciales, de acuerdo con sus registros. Se estableció una muestra equivalente al $20 \%$ del total de las conexiones domésticas, lo que representa 280 usuarios. Esta decisión se tomó en base a Brown, Champ y Boyle (2003), considerando que para una población de tamaño igual a 1.000 , se requieren entre 200 y 300 observaciones para un margen de error de $5 \%$.

En la encuesta se elaboraron sets de elección (SE) los cuales presentaban dos alternativas que combinaban diferentes niveles de las variables consideradas, así como el statu $q u o$. Se listaron seis sets de elección por cada encuesta para determinar el interés del encuestado en cada situación. Se contó con cuatro atributos y varios niveles: tres niveles para el atributo corte de agua, dos niveles para la contaminación del agua, tres niveles para la protección de la quebrada San Alberto y cinco niveles para el pago adicional de la tarifa (se considera como atributo por cuanto es parte de la canasta a presentarse a la población como opción de elección), de los cuales resultan 90 combinaciones ${ }^{2}$.

Los SE se formaron de esta manera:

Tabla 2. Formato de las combinaciones

\begin{tabular}{|l|c|c|c|}
\hline & OPCION A & OPCION B & STATU QUO \\
\hline SE 1 & X3 & X1 & XSQ \\
\hline SE 2: & X2 & X5 & XSQ \\
\hline$\ldots$ & $\ldots$ & $\ldots$ & $\ldots$ \\
\hline SE “n” & X5 & X28 & XSQ \\
\hline
\end{tabular}

Seguidamente, se selecciona el número de SE con que se va a trabajar y se generan las encuestas (de seis SE en este caso). A continuación se muestra un ejemplo:

Se consideraron seis sets de tres opciones cada uno por encuestado. Cada set contenía las opciones A, B y la situación actual. Se utilizaron esquemas gráficos para ayudar a la comprensión del encuestado. Las encuestas fueron realizadas a jefes de familia que no tenían medidores y que pagasen la tarifa de agua potable. Para ello se contó con la lista de usuarios de la EPS y los planos urbanos de la ciudad de Oxapampa, por lo que se tomaron aleatoriamente de la lista 280 nombres.

Para hacer la evaluación de la información obtenida a través de las encuestas realizadas en Oxapampa, se empleó una ficha a la que se le llamó "Tarjeta Ecológica" utilizada en la caracterización y elaboración del formato $23 \times 2 \times 3 \times 5=90$ combinaciones de atributos en sus diferentes niveles. 
Tabla 3. Modelo de Encuesta

\begin{tabular}{|l|l|l|l|}
\hline Opciones Impacto & \multicolumn{1}{|c|}{ OPCIÓN 1 } & \multicolumn{1}{c|}{ OPCIÓN 2 } & \multicolumn{1}{c|}{ OPCIÓN 3 } \\
\hline Corte de servicio de agua & $\begin{array}{l}4-10 \text { horas corte promedio/ } \\
\text { día - 15 días por año }\end{array}$ & Nunca & $\begin{array}{l}\text { 10 horas de corte promedio/ } \\
\text { día - 30 días por año }\end{array}$ \\
\hline $\begin{array}{l}\text { Condición del agua del } \\
\text { río San Alberto }\end{array}$ & $\begin{array}{l}\text { Río San Alberto sin } \\
\text { contaminación. }\end{array}$ & $\begin{array}{l}\text { Río San } \\
\text { Alberto sin } \\
\text { contaminación. }\end{array}$ & $\begin{array}{l}\text { Rio San Alberto } \\
\text { contaminado. }\end{array}$ \\
\hline $\begin{array}{l}\text { Protección de la cuenca } \\
\text { San Alberto }\end{array}$ & $\begin{array}{l}50 \% \text { de las quemas que } \\
\text { hoy se realizan. }\end{array}$ & $\begin{array}{l}\text { No se realizan } \\
\text { quemas. }\end{array}$ & $\begin{array}{l}\text { Mismo número de } \\
\text { quemas que hoy }\end{array}$ \\
\hline $\begin{array}{l}\text { Tarifa doméstica mensual } \\
\text { de agua }\end{array}$ & S/. 8,90 + S/.0,00 & $\begin{array}{l}\text { S/. 8,90 }+ \text { S/. } \\
6,00\end{array}$ & S/. 8,90 + S/. 0,00 \\
\hline \multicolumn{1}{|c|}{ Su Opción } & & & \\
\hline
\end{tabular}

de encuesta, a través de la cual se identifican tres niveles: nivel 1 (statu quo o situación actual), nivel 2 (nivel medio) y nivel 3 (nivel óptimo), además de los niveles 4 y 5 para la tarifa planteada, para las variables consideradas como determinantes en la contaminación de la quebrada San Alberto, tal como se muestra la Tabla 4.

El procesamiento de las encuestas se realizó empleando el
El resto de columnas se completó con los datos obtenidos directamente de las encuestas.

El procedimiento de estimación estadística para hallar los parámetros de utilidad del modelo de elección, se realizó mediante la estimación de Máxima Verosimilitud (MLE), el mismo que puede ser extendido fácilmente a situaciones en las que una población está caracterizada por más de un

Tabla 4. Niveles de atributo

\begin{tabular}{|c|c|c|c|c|c|}
\hline Atributos & Nivel 1: Statu Quo & Nivel 2 & Nivel 3 & Nivel 4 & Nivel 5 \\
\hline $\begin{array}{l}\text { Cortes (interrupciones de } \\
\text { servicio de agua potable) }\end{array}$ & $\begin{array}{c}10 \text { horas de corte } \\
\text { promedio/día }-30 \\
\text { días por año }\end{array}$ & $\begin{array}{l}\text { 4-10 horas de corte } \\
\text { promedio/día }-15 \\
\text { días por año }\end{array}$ & nunca & & \\
\hline Agua (calidad del agua del río) & $\begin{array}{c}\text { Rio San Alberto } \\
\text { contaminado }\end{array}$ & $\begin{array}{l}\text { Rio San Alberto } \\
\text { sin contaminación }\end{array}$ & & & \\
\hline $\begin{array}{c}\text { Quemas (protección de la } \\
\text { cuenca - quemas del bosque) }\end{array}$ & $\begin{array}{l}\text { Mismo número de } \\
\text { quemas }\end{array}$ & $\begin{array}{c}50 \% \text { de las } \\
\text { quemas que hoy se } \\
\text { realizan }\end{array}$ & $\begin{array}{l}\text { No se } \\
\text { realizan } \\
\text { quemas }\end{array}$ & & \\
\hline $\begin{array}{c}\text { Tarifa (para agua potable } \\
\text { mensual) }\end{array}$ & $\mathrm{S} / 8,90+0$ & $\mathrm{~S} / 8,90+2,00$ & $\begin{array}{c}\mathrm{S} / 8,90+ \\
4,00\end{array}$ & $\begin{array}{c}\mathrm{S} / 8,90+ \\
6,00\end{array}$ & $\begin{array}{l}\mathrm{S} / 8,90+ \\
\quad 8,00\end{array}$ \\
\hline
\end{tabular}

modelo Logit, a través del software NLogit 3.0. La data obtenida fue procesada por individuos donde la respuesta a obtenerse corresponde a la elección de la opción. Para llenar las columnas de los sets, se emplearon los números 1,2 y 3 para identificar el número de opción elegida por set y se escribió “-999" cuando los encuestados no respondieron nada. Posteriormente, se ordenó la información adicionando al cuadro anterior, los códigos de efecto (effect codes) y los códigos para opción (codes for option), como se muestra en el Anexo $\mathrm{N}^{\mathrm{o}} 1$.

Se emplearon 18 líneas por encuesta, desagregadas en seis sets de tres opciones cada uno. Los códigos de efecto (effect codes) son los valores "1" para el nivel 1, "0" para el nivel 2 y "-1" para el statu quo o nivel actual, asignados a los factores para identificar el nivel al que pertenecen, dentro de la combinación representativa de cada opción. En la columna "Opción elegida" se colocó " 0 " para las opciones no electas y "1" para la opción elegida de cada set. parámetro. El modelo básico de elección consistente con los supuestos dados es llamado la Elección Condicional Logit o Logit Multinomial (MNL).

Se plantearon las siguientes dos formulaciones: el modelo básico y una que adicionalmente contiene algunas variables socio-demográficas importantes (SDVs).

\section{Resultados y discusión}

La primera estimación que se realizó fue:

$\mathrm{Ujt}=\beta \mathrm{jt}+\beta \mathrm{jt}($ corte 1$) \mathrm{jt}+\beta \mathrm{jt}($ corte 2$) \mathrm{jt}+\beta \mathrm{jt}($ agua 1$) \mathrm{jt}+\beta \mathrm{jt}$ (quema1)jt $+\beta \mathrm{jt}($ quema2)jt $+\beta \mathrm{jt}($ tarifa $) \mathrm{jt}+\xi \mathrm{jt}$

En la Tabla 5 se encuentran los resultados de este primer modelo donde se aprecia únicamente el modelo con las variables significativas. El significado del número al lado de la variable significa lo siguiente: cuando tiene 1 se elimina por completo el problema y cuando se utiliza el número 2 se plantea una solución intermedia. Este modelo presenta un $\mathrm{R}^{2}$ de 0,347 , manteniendo los signos esperados 
de los coeficientes de las variables consideradas y su nivel de significancia. La salida completa se encuentra en el Anexo 2.

La segunda estimación fue:

Tabla 5. Resultado del modelo básico reducido

\begin{tabular}{|c|c|c|c|c|}
\hline Variable & Coeficiente & $\begin{array}{l}\text { Error } \\
\text { estándar }\end{array}$ & $\mathrm{b} / \mathrm{ES}$ & $\mathrm{P}((\mathrm{z})>\mathrm{z})$ \\
\hline ASC3 & $-0,545$ & 0,145 & $-3,757$ & 0,0002 \\
\hline Corte1 & 0,286 & 0,048 & 5,998 & 0,0000 \\
\hline Agua1 & 0,844 & 0,044 & 19,194 & 0,0000 \\
\hline Quema1 & 0,531 & 0,049 & 10,765 & 0,0000 \\
\hline Tarifa & $-0,051$ & 0,014 & $-3,722$ & 0,0002 \\
\hline
\end{tabular}

$\mathrm{Ujt}=\beta \mathrm{jt}+\beta \mathrm{jt}($ corte 1$) \mathrm{jt}+\beta \mathrm{jt}(\operatorname{corte} 2) \mathrm{jt}+\beta \mathrm{jt}($ agua 1$) \mathrm{jt}+\beta \mathrm{jt}$ (quema1)jt $+\beta \mathrm{jt}$ (quema2)jt $+\beta \mathrm{jt}($ tarifa $) j \mathrm{t}+\beta \mathrm{jt}($ visitasX) $\mathrm{jt}+\beta \mathrm{jt}(\mathrm{cual} \mathrm{X}) \mathrm{jt}+\beta \mathrm{jt}(\mathrm{tiempoX}) \mathrm{jt}+\beta \mathrm{jt}($ persX)jt $+\beta \mathrm{jt}$ $($ pers 18X)jt $+\beta \mathrm{jt}($ edadX)jt $+\beta \mathrm{jt}($ generoX)jt $+\beta \mathrm{jt}($ instruX $)$ $\mathrm{jt}+\beta \mathrm{jt}($ ingresoX)jt $+\xi \mathrm{jt}$

En este caso la función 2 contiene cuatro variables sociodemográficas (INGRESOX, GENEROX, EDADX, y PERS18X - cuando la familia tiene personas mayores de 18 años). La idea, en esta ocasión, es relacionar estas variables con la DAP. El resultado, tomando en consideración las variables significativas, se presenta en la Tabla 6 . El $\mathrm{R}^{2}$ de esta es de 0,36, la estimación completa está en el Anexo 3.

Tabla 6. Resultado del modelo con variables sociodemográficas

\begin{tabular}{|c|c|c|c|c|}
\hline Variable & Coeficiente & $\begin{array}{l}\text { Error } \\
\text { estándar }\end{array}$ & $\mathrm{b} / \mathrm{ES}$ & $\mathrm{P}((\mathrm{z})>\mathrm{z})$ \\
\hline ASC3 & 1,008 & 0,419 & 2,405 & 0,0162 \\
\hline Corte1 & 0,281 & 0,048 & 5,849 & 0,0000 \\
\hline Agua1 & 0,849 & 0,044 & 19,109 & 0,0000 \\
\hline Quema1 & 0,533 & 0,050 & 10,716 & 0,0000 \\
\hline Tarifa & $-0,051$ & 0,014 & $-3,706$ & 0,0002 \\
\hline IngresoX & 0,0003 & 0,0002 & 2,167 & 0,0302 \\
\hline GeneroX & $-0,810$ & 0,214 & $-3,796$ & 0,0001 \\
\hline Pers18X & 0,275 & 0,092 & 2,983 & 0,003 \\
\hline
\end{tabular}

Determinación del valor del servicio ambiental de provisión de agua para la población de Oxapampa.

En la Tabla 7 se encuentran los resultados de la disposición a pagar según las dos estimaciones realizadas y que señalan que los usuarios del agua en Oxapampa están dispuestos a pagar un costo adicional a la tarifa mensual.

Dado el $\mathrm{R}^{2}$ ajustado del segundo modelo, luego de eliminar las variables no significativas, se deduce que este es el modelo a elegir. En la Tabla 7 se presentan las DAP de la población por las diferentes variables analizadas. Estas se obtuvieron de la razón entre el coeficiente de la variable en estudio y el valor de la tarifa multiplicado por (-1).
Tabla 7. Determinación de la DAP considerando solo las variables significativas en nuevos soles

\begin{tabular}{|c|c|c|c|c|}
\hline \multirow{2}{*}{ Estimación } & \multirow{2}{*}{$\mathrm{R}^{2}$} & \multirow{2}{*}{$\begin{array}{c}\text { DAP por el } \\
\text { agua }\end{array}$} & \multicolumn{2}{|c|}{ Otros DAP } \\
\cline { 4 - 5 } & & & Atributo & $\mathrm{DAP}$ \\
\hline \multirow{2}{*}{ 1ra estimación } & \multirow{2}{*}{0,347} & \multirow{2}{*}{16,65} & Corte 1 & 5,65 \\
\cline { 4 - 5 } & & & Quema1 & 10,47 \\
\hline \multirow{2}{*}{ 2da estimación } & \multirow{2}{*}{0,363} & \multirow{2}{*}{16,66} & Corte 1 & 5,51 \\
\cline { 4 - 5 } & & & Quema1 & 10,47 \\
\hline
\end{tabular}

De los resultados obtenidos, se puede deducir que no hay evidencia que la población tenga disponibilidad a pagar por obtener el $50 \%$ del beneficio, frente a la eliminación total de los problemas de interrupciones de servicio de agua y quemas de bosques. Esto refleja la falta de interés por soluciones parciales, lo que a su vez también puede ser explicado porque los usuarios del servicio no consideran entre sus opciones válidas escenarios intermedios.

Desde el punto de vista económico la calidad del agua de San Alberto es la variable más importante para la población y debe ser considerada una prioridad en la lista de soluciones. En segundo lugar se tiene a las quemas en la zona de amortiguamiento, con una importancia media.

\section{Conclusiones}

La DAP calculada en base al modelo de experimentos de elección es igual a S/. 16,66 nuevos soles, el cual es bastante mayor al valor que actualmente se paga por la tarifa de agua. Este valor se puede entender en el contexto de las interrupciones en el servicio que son una constante en el poblador de Oxapampa, por lo que no debe de sorprender que el monto determinado esté por encima de la tarifa actual. Igualmente, es importante mencionar que los pobladores prefieren soluciones totales a medidas parciales a ser implementadas, es decir las estimaciones mostraron que cuando las personas se enfrentan a opciones donde se propone una solución intermedia esta no es igualmente apreciada por los consumidores, como en el caso de la solución total. Esto es importante en la formulación de políticas, dado que las mismas solo serán aceptadas por la población si las propuestas son integrales en su solución, no debiendo proponerse esquemas intermedios.

Además de ello, se debe resaltar que el método por sí mismo intenta incorporar en la determinación su comparación con otras variables, lo cual tiene la ventaja de cumplir con un requisito de la teoría de las preferencias, donde se sostiene la posibilidad de sustituir un bien por otro, a diferencia de la valoración contingente que tiene este tipo de sesgo.

El valor encontrado podría servir como base para un incremento en las tarifas de agua potable. Habiéndose determinado un monto que es el doble de la tarifa actual, hay un espacio amplio para esta posible alza, pudiéndose realizar la misma a partir de las características socioeconómicas de la población.

Una tarea pendiente es la de determinar cuál sería la tarifa apropiada si además de los problemas presentados se mencionase la calidad del agua potable. Esta consideración no se ha trabajado, en este caso, pero la evidencia señala 
que si se le incluye, el valor del recurso deberá ser mayor.

\section{Literatura citada}

Brown, T.; Champ, P. y Boyle, K. 2003. A Primer on Nonmarket Valuation. USA: Kluwer Academis Publishers. Colombo, S. (2004). Valoración y análisis económico de impactos ambientales en procesos erosivos: aplicación de los métodos de valoración contingente y experimento de elección en la cuenca del Alto Genil. Cuadernos Geográficos, 34, 223-232.

Hanley, N.; Wright, R.E. y Alvarez-Farizo, B. 2006. Estimating the economic value of improvements in river ecology using choice experiments: an application to the water framework directive. Journal of Environmental Management, 78(2), 183-93.

Hanley, N.; Wright, R. y Adamowicz, V. 1998. Using Choice Experiments to Value the Environment. Environmental and Resource Economics, 11(3-4), 413428.

Hatton, M,D.; Barnes, M.; Bennett, J.; Morrison, M. y Young, M. 2005. Using a Choice Modelling Approach for Customer Service Standards in Urban Water. Journal of American. Water Resources Association, 41, 719-728.

Hensher, D.; Rose, J. y Greene, W. 2005. Applied Choice Analysis. Reino Unido: Cambridge University Press.

Hensher, D.; Shore, N. y Train, K. 2005. Households' Willingness to Pay for Water Service Attributes. Environmental \& Resource Economics, 32 (4), 509-531.

United Nations Environment Programme [UNEP]. 2004. The use of economic instruments in environmental policy: opportunities and challenges. UNEP.

Anexo: 


\begin{tabular}{|c|c|c|}
\hline \multicolumn{3}{|c|}{ Anexo $N^{D} 1:$ Variables empleadas en el procesamiento de la información } \\
\hline Código & Nombre Completo & Explicación \\
\hline id & $N^{\circ}$ encuesta & Número de encuestados (encuesta) \\
\hline cset & cset & Número de opciones por choice sel \\
\hline set & Set & Choice set $(1,2,3,4,5$, or 6$)$ \\
\hline opcion & Opción & Opción (1=A, 2=B, 3=SQ=status quo) \\
\hline corte1 & Corte1 & $\begin{array}{l}\text { Effects code para los cortes de servicio }(-1=S Q \text {, } \\
\left.0=\text { nivel } 1,1=\text { nivel } 2 \text { ) (Ver Anexo Cuadro } N^{\circ} 08\right)\end{array}$ \\
\hline corte2 & Corte2 & $\begin{array}{l}\text { Effects code para los cortes de servicio }(-1=S Q \text {, } \\
1=\text { level } 1,0=\text { nivel } 2)\left(\text { Ver Anexo Cuadro } N^{\circ} 08\right)\end{array}$ \\
\hline agua 1 & Agua1 & $\begin{array}{l}\text { Effects code para la calidad del agua de la quebrada } \\
\left.(-1=S Q, 1=\text { nivel } 1) \text { Ver Anexo Cuadro } N^{\circ} 08\right)\end{array}$ \\
\hline quema1 & Quema1 & $\begin{array}{l}\text { Effects code para la queme de bosques }(-1=S Q \text {, } \\
\left.0=\text { level } 1,1=\text { nivel 2) (Ver Anexo Cuadro } N^{\circ} 08\right)\end{array}$ \\
\hline quema2 & Quema2 & $\begin{array}{l}\text { Effects code para la queme de bosques }(-1=S Q \text {, } \\
\left.1=\text { nivel } 1,0=\text { nivel 2) (Ver Anexo Cuadro } N^{\circ} 08\right)\end{array}$ \\
\hline tarifa & Tarifa & $\begin{array}{l}0=S Q, 2,4,6,8 \text { (aumento de la tasa en Nuevos Soles } \\
\text { por mes) Ver Anexo Cuadro } N^{\circ} 08 \text { ) }\end{array}$ \\
\hline choice & Opción elegida & $0=0$ ción no seleccionada, $1=$ opción seleccionada \\
\hline visitas & $\begin{array}{l}\text { Visitas a la Quebrada San } \\
\text { Alberto }\end{array}$ & $\begin{array}{l}\text { Número de visitas a la Cuenca San Alberto (ver } \\
\text { Cuadro } N^{\circ} \text { 6-1 en Anexo } N^{\circ} 6 \text { ) }\end{array}$ \\
\hline calidad & $\begin{array}{l}\text { Calidad del Agua de San } \\
\text { Alberto }\end{array}$ & $\begin{array}{l}\text { Calidad del agua percibida en la quebrada San } \\
\text { Alberto (ver Cuadro } N^{\circ} 6-2 \text { en Anexo } N^{\circ} 6 \text { ) }\end{array}$ \\
\hline tiempo & Tiempo que vive en área & Años viviendo en el área \\
\hline person & $N^{\circ}$ personas en casa & Número de personas viviendo en la casa. \\
\hline person 18 & $\begin{array}{l}N^{\circ} \text { personas menores de } \\
18 \text { años }\end{array}$ & Número de personas menores de 18 años de edad. \\
\hline occup & Ocupación & Occupación (ver Cuadro $N^{\circ} 6-5$ en Anexo $N^{\circ} 6$ ) \\
\hline edad & Edad & Edad (en años). \\
\hline genero & Género & Género ( $0=$ mujer, $1=$ hombre $)$ \\
\hline instru & Instrucción & Nivel de educación (ver Cuadro $N^{\circ}$ 6-3 en Anexo $N^{\circ} 6$ ) \\
\hline ingreso & Ingreso Familiar & $\begin{array}{l}\text { Ingreso familiar mensual (ver Cuadro } N^{\circ} 6-4 \text { en } \\
\text { Anexo } N^{\circ} 6 \text { ) }\end{array}$ \\
\hline cortes50 & \begin{tabular}{|l} 
Reducir Ser de Agua al \\
$50 \%$
\end{tabular} & $\begin{array}{l}\text { Escala de 7-puntos de acuerdo (ver Cuadro } N^{\circ} 6-6 \text { en } \\
\text { Anexo } N^{\circ} 6 \text { ) }\end{array}$ \\
\hline cortes 0 & Eliminar cortes de agua & $\begin{array}{l}\text { Escala de } 7 \text {-puntos de acuerdo (ver Cuadro } N^{\circ} 6-6 \text { en } \\
\text { Anexo } N^{\circ} 6 \text { ) }\end{array}$ \\
\hline quimic50 & $\begin{array}{l}\text { Reducir uso agroquímico } \\
\text { al } 50 \%\end{array}$ & $\begin{array}{l}\text { Escala de } 7 \text {-puntos de acuerdo (ver Cuadro } N^{\circ} 6-6 \text { en } \\
\text { Anexo } N^{\circ} 6 \text { ) }\end{array}$ \\
\hline quimico & Eliminar uso agroquímico & $\begin{array}{l}\text { Escala de } 7 \text {-puntos de acuerdo (ver Cuadro } N^{\circ} 6-6 \\
\text { en Anexo } N^{\circ} 6 \text { ) }\end{array}$ \\
\hline quemas50 & Reducir $n^{\circ}$ quemas al $50 \%$ & $\begin{array}{l}\text { Escala de 7-puntos de acuerdo (ver Cuadro } N^{\circ} 6-6 \text { en } \\
\text { Anexo } N^{\circ} 6 \text { ) }\end{array}$ \\
\hline quemaso & Eliminar todas las quemas & $\begin{array}{l}\text { Escala de } 7 \text {-puntos de acuerdo (ver Cuadro } N^{\circ} 6-6 \text { en } \\
\text { Anexo } N^{\circ} 6 \text { ) }\end{array}$ \\
\hline
\end{tabular}




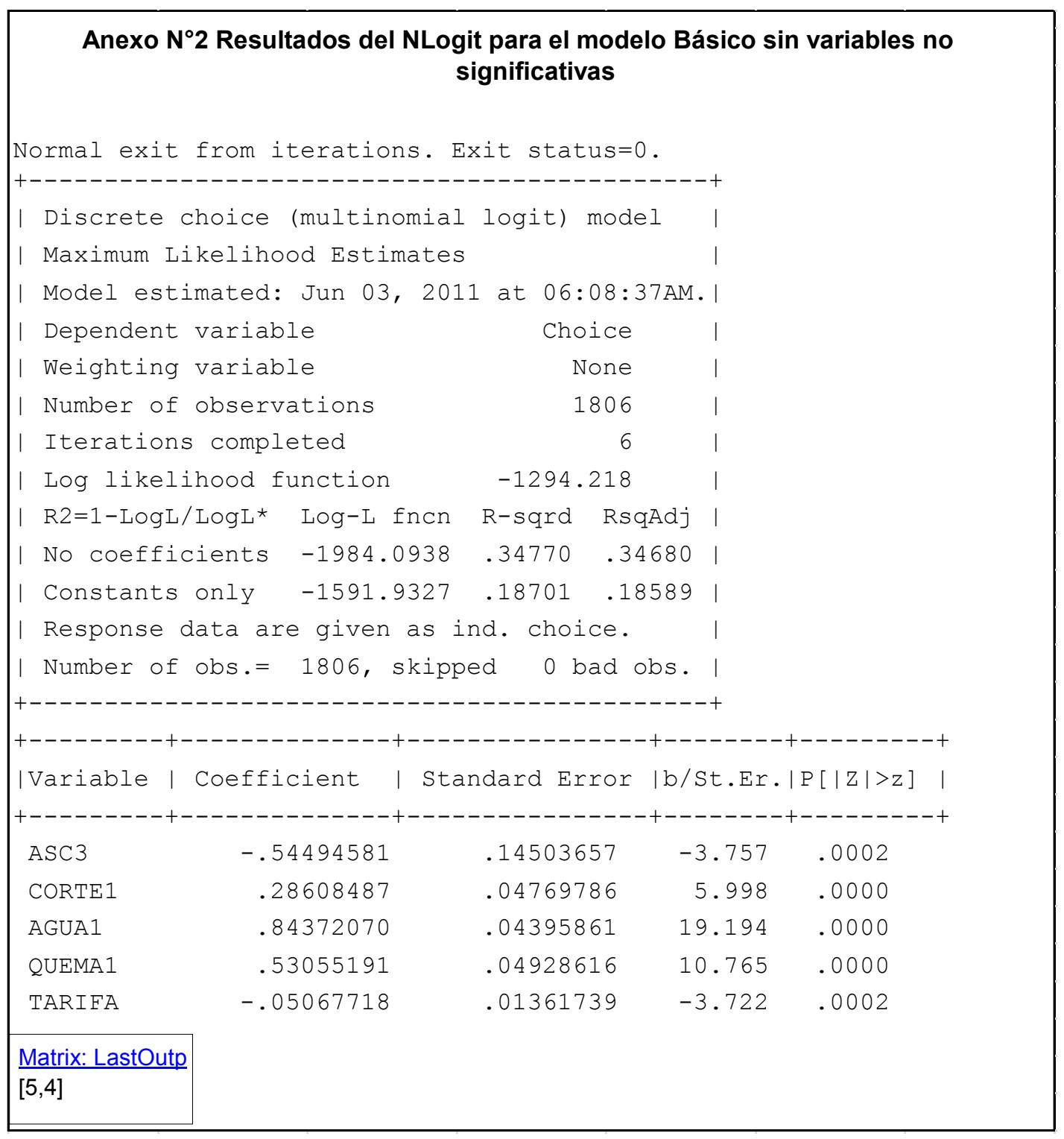




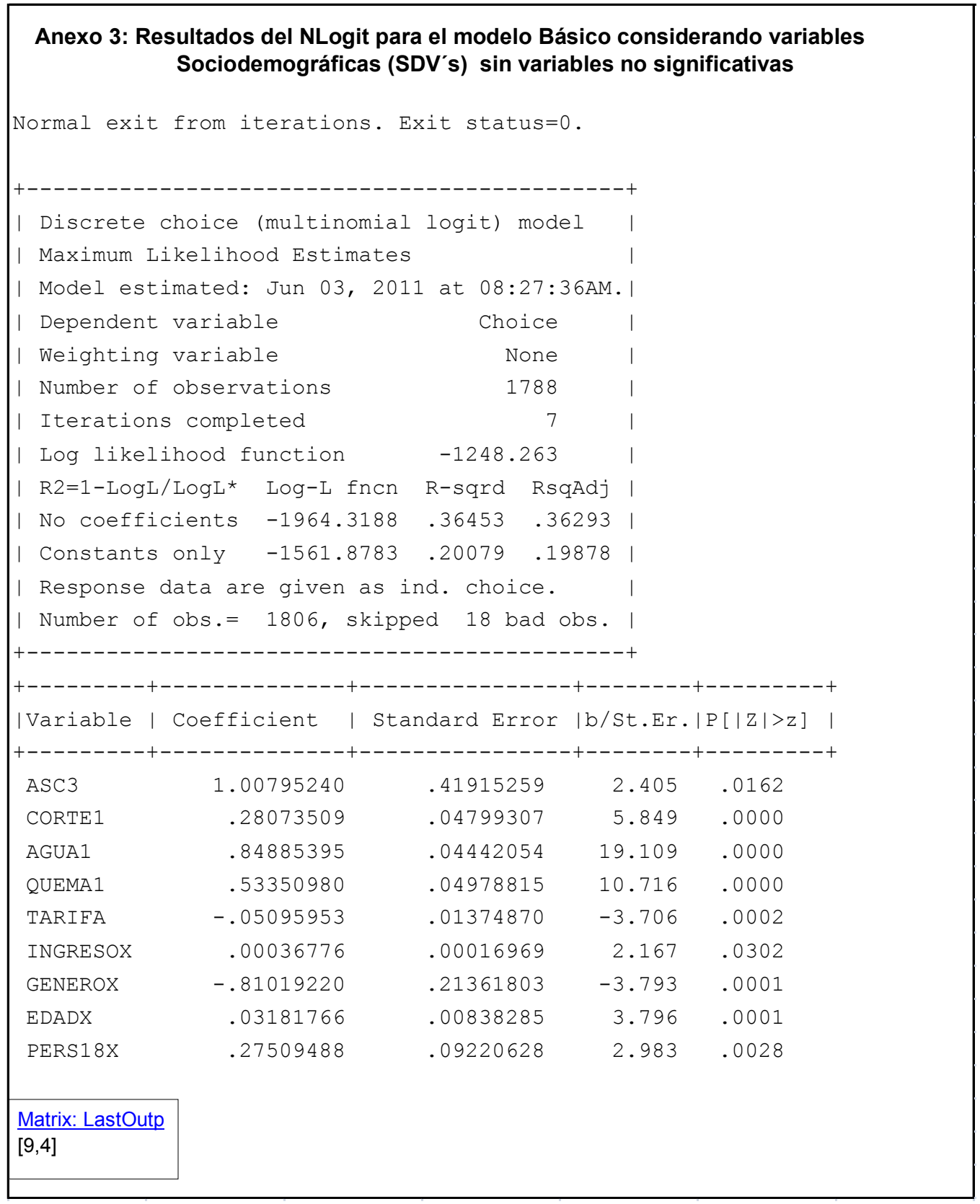

\begin{tabular}{|l|l|l|}
\hline Código & Nombre completo & Explicación \\
\hline id & $\mathrm{N}^{\circ}$ encuesta & Número de encuestados \\
\hline cset & cset & Número de opciones por choice set \\
\hline set & set & choice set $(1,2,3,4,5$, o 6$)$ \\
\hline Opción & Opción & Opción $(1=\mathrm{A}, 2\} 0 \mathrm{~B}, 3=\mathrm{SQ}$ (status quo) \\
\hline corte1 & Corte1 & \\
\hline
\end{tabular}

